Ann. Zootech., I979, 28 (3), 26I-269.

\title{
Influence du niveau énergétique et de la granulation du régime sur les performances de croissance et l'engraissement du pintadeau. Comparaison avec le poulet
}

\author{
J.-C. BLUM et B. LECLERCQ \\ Station de Recherches avicoles \\ I.N.R.A., Centre de Tours-Nouzilly \\ 37380 Monnaie (France)
}

\section{Résumé}

Dans une expérience factorielle, on constitue 4 lots en fonction du niveau énergétique ( 2 goo à 3240 Kcal E.M. $/ \mathrm{kg}$ ) et du mode de présentation (farine ou granulés) de l'aliment.

Chaque lot comprend 6 cases de poulets ( 53 poulets par case), soumis à l'expérience de 7 à 56 jours d'âge et 6 cases de pintadeaux ( 62 pintadeaux par case), testés de 7 à 84 et 98 jours.

Chez le poulet, on confirme les effets bénéfiques de la granulation, effets plus marqués lorsque les performances (gain de poids, indice de consommation) ont été quelque peu compromises par la distribution de régimes à niveau énergétique bas. Chez le pintadeau, la consommation alimentaire est la même dans tous les lots à l'âge de 28 jours. Mais, par la suite, l'animal règle sa consommation en fonction du niveau énergétique de sorte que l'influence des 2 facteurs étudiés sur le gain de poids est plus faible que chez le poulet. A l'âge de I 2 semaines, la granulation n'a pas eu d'effet significatif sur le poids vif du pintadeau pour l'apport énergétique élevé, elle n'a eu qu'un faible effet $(+30 \mathrm{~g})$ pour l'apport énergétique bas.

La dissection des carcasses montre que les facteurs qui augmentent le poids vif des pintadeaux âgés de $\mathrm{I}_{2}$ et $\mathrm{I} 4$ semaines (apport énergétique élevé, maturité sexuelie chez les femelles) agissent exclusivement par accroissement des dépôts adipeux.

\section{Introduction}

CAUChard (I97I) rapporte que le pintadeau a un besoin énergétique élevé; il recommande de présenter l'aliment sous forme de granulés. En cherchant à préciser les besoins pendant la croissance, nous avons montré que le pintadeau était plutôt moins sensible que le poulet aux variations de l'apport énergétique (BLUM, GUILlAUME et LECLERCQ, I975). I1 restait à étudier 1'influence de la granulation, objet de la présente expérience où, une fois de plus, nous comparons poulet et pintadeau. Pour tenir compte de l'interaction connue chez le poulet 


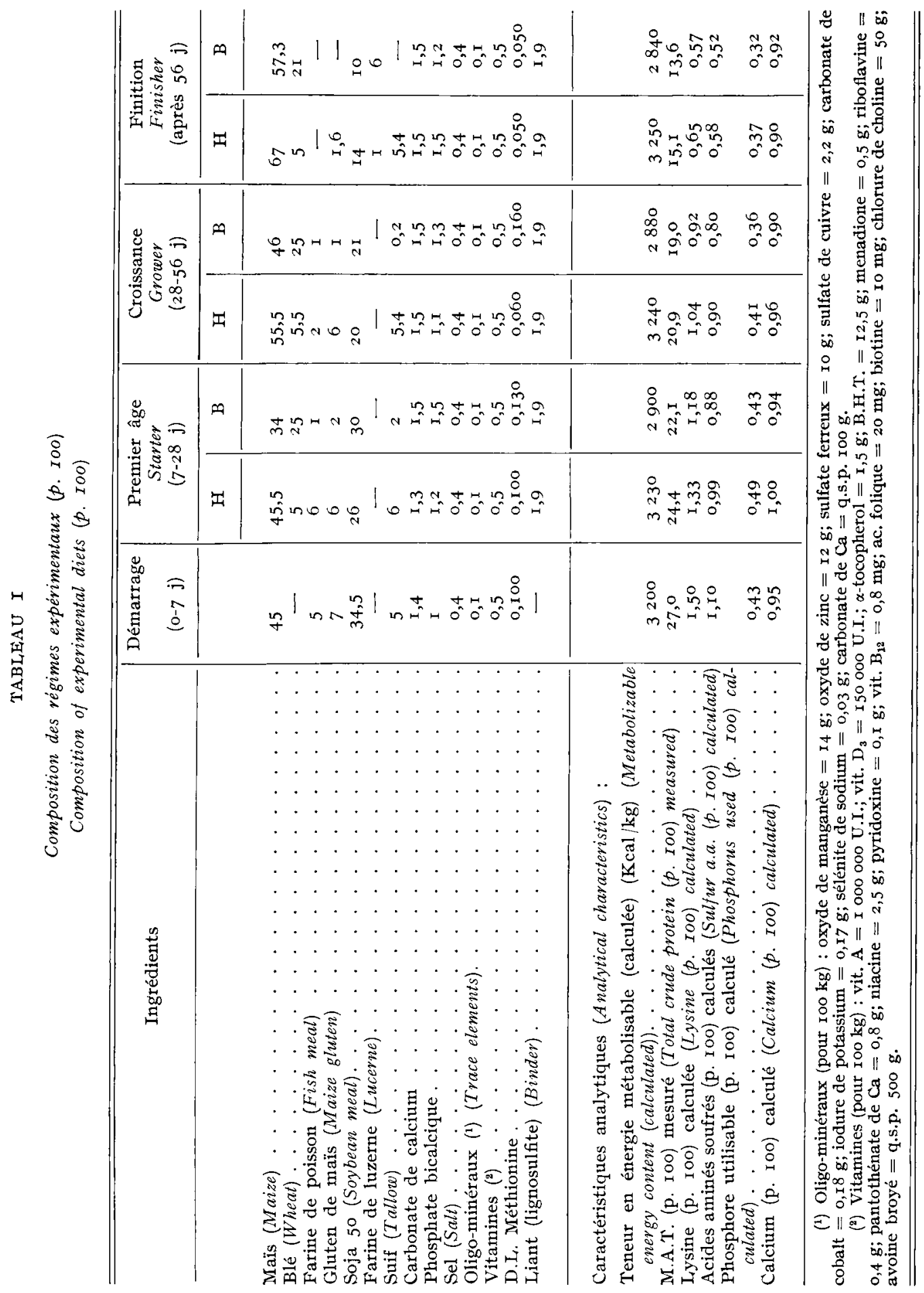


entre apport énergétique et granulation (CALET, I965), 2 types de régimes sont distribués : régimes $H$ à concentration énergétique élevée, régimes $B$ à concentration énergétique basse. Nous profitons également de cet essai pour rechercher l'importance de l'engraissement chez le pintadeau à la fin de la période de croissance.

\section{Matériel et méthodes}

Les animaux sont élevés au sol sur litière de copeaux dans un poulailler comprenant 48 cases de $6 \mathrm{~m}^{2}$ chacune. Les pintadeaux éclos à la Station (mélange des sexes, souches BEGHIN et GALOR) sont placés dans 24 de ces cases à raison de 62 pintadeaux par case. Les poussins (HubBARD de sexe mâle) occupent les 24 autres cases à raison de 53 poussins par case. Les conditions d'éclairement et de chauffage ont été les mêmes que précédemment (BLum, Guillaume et LECIERCQ, I975).

La composition des régimes est rapportée dans le tableau $\mathbf{I}$. Pintadeaux et poulets ingèrent les mêmes aliments aux mêmes âges. Le régime démarrage est distribué à tous les animaux de 0 à 7 jours sous forme de farine. Les autres régimes ont un niveau énergétique haut $(\mathrm{H})$ ou bas $(\mathrm{B})$; ils sont présentés soit en farine, soit en granulés à 6 cases de poulets et à 6 cases de pintadeaux. A chaque âge correspond un type de régime : "I er âge " de 7 à 28 jours, "croissance ", de 28 à 56 jours, " finition " pour les pintadeaux car ils sont conservés au delà de 56 jours.

Les poulets sont sacrifiés à 1'âge de 8 semaines, les pintadeaux à $\mathrm{I} 2$ semaines, à l'exception de 4 cases alimentées avec les régimes "finition " granulés ( 2 cases sur régime $\mathrm{H}, 2$ cases sur régime $B$ ) jusqu'à l'âge de I4 semaines.

On enregistre le poids vif des animaux et leur consommation d'aliment à 4 , 8 , I2 et I4 semaines. De plus, à I 2 et I 4 semaines, on choisit 40 pintadeaux représentatifs de chacun des lots $\mathrm{H}$ et $\mathrm{B}$ nourris sur régimes granulés. Après sacrifice de ces pintadeaux, on procède à une dissection des carcasses. Le gras abdominal est séparé selon la technique précédemment décrite (BLUM et LECLERCQ, I978); en même temps, on identifie le sexe des pintadeaux.

La signification staristique des résultats est établie par analyse de variance. Les comparaisons de moyennes sont effectuées grâce au test de NEwMAN et KEULS (KEULS, I952).

\section{Résultats}

A 1'âge de 4 semaines (tabl. 2), la granulation améliore nettement la croissance et l'indice de consommation des poulets nourris avec le régime à niveau énergétique bas (B); par contre, avec le régime $\mathrm{H}$, farine et granulés assurent les mêmes performances. Chez le pintadeau, la granulation est sans effet; seul le taux énergétique intervient en accélérant légèrement la croissance et en diminuant l'indice de consommation; la quantité d'aliment ingérée est la même pour tous les régimes.

A l'âge de 8 semaines (tabl. 3), granulation et accroissement du taux énergétique ont tous les deux un effet bénéfique sur les performances du poulet. Cependant, l'influence de la granulation est plus importante dans le cas du régime à taux énergétique bas. Chez le pintadeau, on confirme les observations faites à 
TABLEAU 2

Influence du taux énergétique et de la granulation des régimes sur les performances de croissance à l'áge de 4 semaines

Infuence of energy level and pelleting of diets on growth performances of chicken and guinea fowl at 4 weeks of age

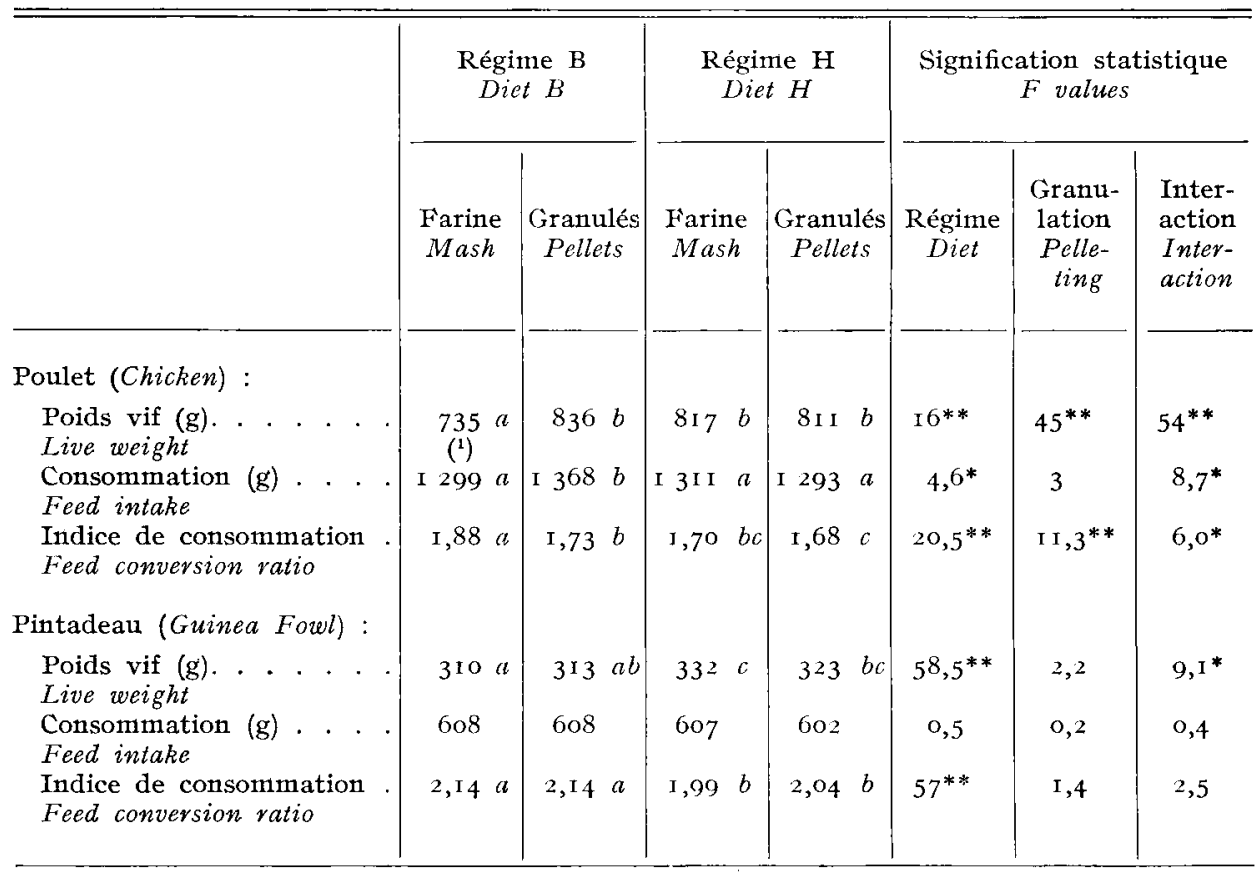

(1) Pour chaque espèce, les performances qui ne sont pas suivies de la même lettre diffèrent significativement entre elles ( $\mathbf{P}<0,05$ ) (For each species, values that are not followed by the same letter are significantly different).

* Significatif au seuil de 5 p. Ioo (Significant at the threshold of 5 p. Ioo).

** Significatif au seuil de $I$ p. Ioo (Significant at the threshold of $I p$. Ioo).

4 semaines; toutefois, l'apport énergétique élevé n'accélère plus la croissance, les animaux sur régime $\mathrm{H}$ conservent leur avance (poids vif plus élevé) mais ne réalisent pas un gain de poids supérieur de 4 à 8 semaines. La consommation et l'indice sont diminués par l'accroissement de l'apport énergétique. Presque sans effet, la granulation tend tout de même à accroître la consommation entre 4 et 8 semaines chez les pintadeaux consommant le régime $B$.

Chez le pintadeau âgé de $I 2$ semaines (tabl. 4) des similitudes apparaissent avec le poulet de 8 semaines. Dans les deux espèces, granulation et niveau énergétique ont accéléré la croissance, l'influence de la granulation se manifestant surtout pour un apport énergétique réduit (régime $B$ ). Les deux facteurs alimentaires améliorent également 1 indice pendant la dernière période $(8$ à $\mathrm{r} 2$ semaines pour le pintadeau). La différence entre les deux espèces porte sur l'importance des réactions. En prenant le régime $B$ en farine comme référence, on constate que la granulation et l'accroissement du niveau énergétique ont tous deux aug- 


\section{TABLEAU 3}

Influence du taux énergétique et de la granulation des régimes sur les performances de croissance à l'âge de 8 semaines

Infuence of energy level and pelleting of diets on growth performances of chicken and guinea fowl at 8 weeks of age

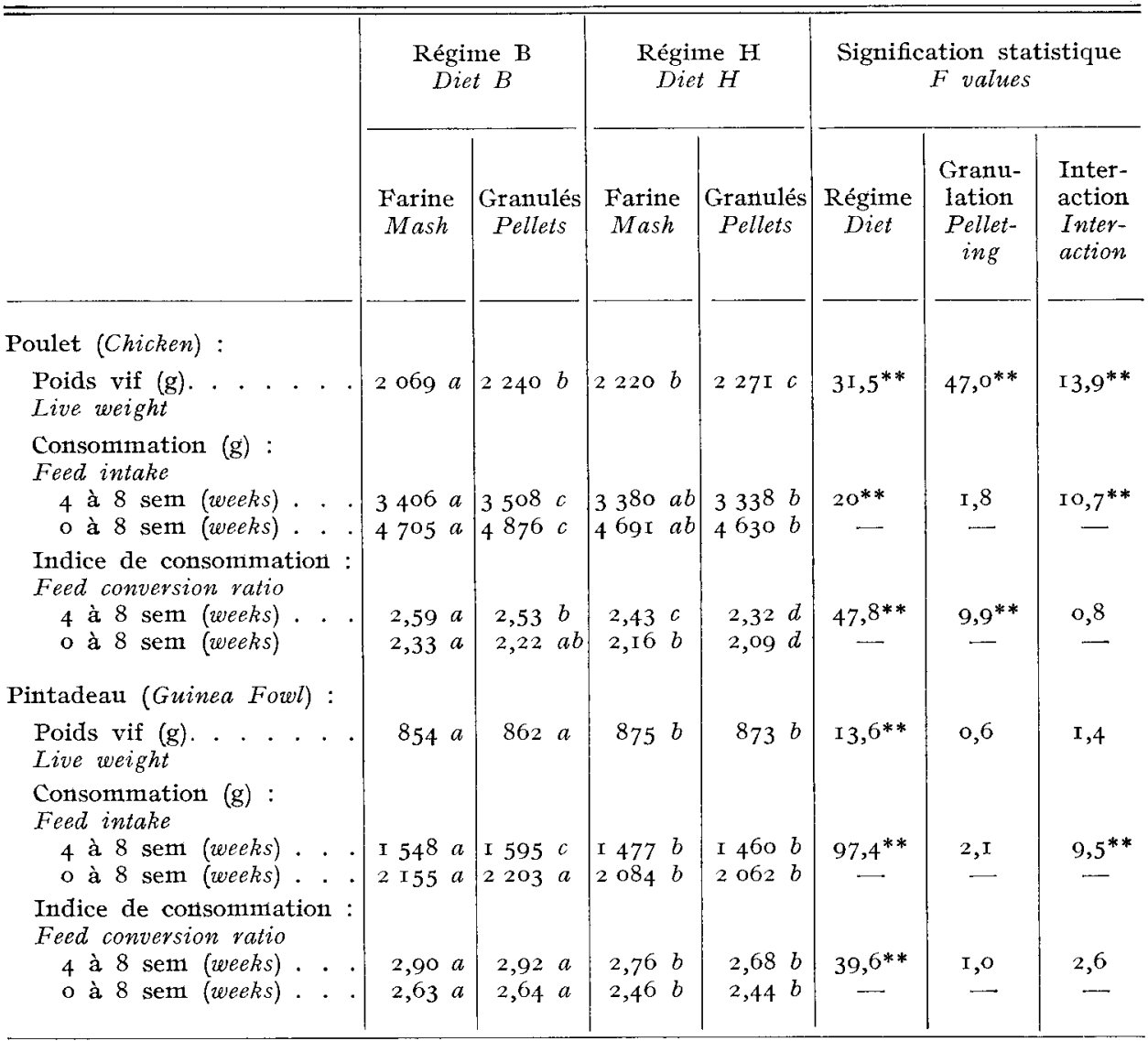

(1) Cf. tableaut 2 .

menté le poids vif final, de plus de 8 p. Ioo chez le poulet contre respectivement 2 et 4 p. roo chez le pintadeau. La prolongation de l'expérience avec les régimes granulés montre que l'influence du niveau énergétique sur le poids vif demeure positive à 1'âge de $\mathrm{I} 4$ semaines. Le ralentissement de la croissance du pintadeau a pour conséquence une augmentation importante de l'indice de consommation.

L'examen des carcasses (tabl. 5) permet de mieux analyser l'influence des régimes en fonction du sexe et de 1'âge des pintadeaux. A I2 semaines, mâles et femelles ont des poids vifs sensiblement identiques; cependant, la consommation d'un aliment très énergétique (régime $\mathrm{H}$ ) accroît surtout le poids des femelles. A I4 semaines, les femelles sont nettement plus lourdes que les mâles, 
TABLEAU 4

Infuence des régimes sur les performances de croissance du pintadeau aux ages de I2 et I4 semaines

Infuence of experimental diets on growth performances of guinea fowl at $\mathrm{I} 2$ and $\mathrm{I} 4 \mathrm{\text {weeks }}$

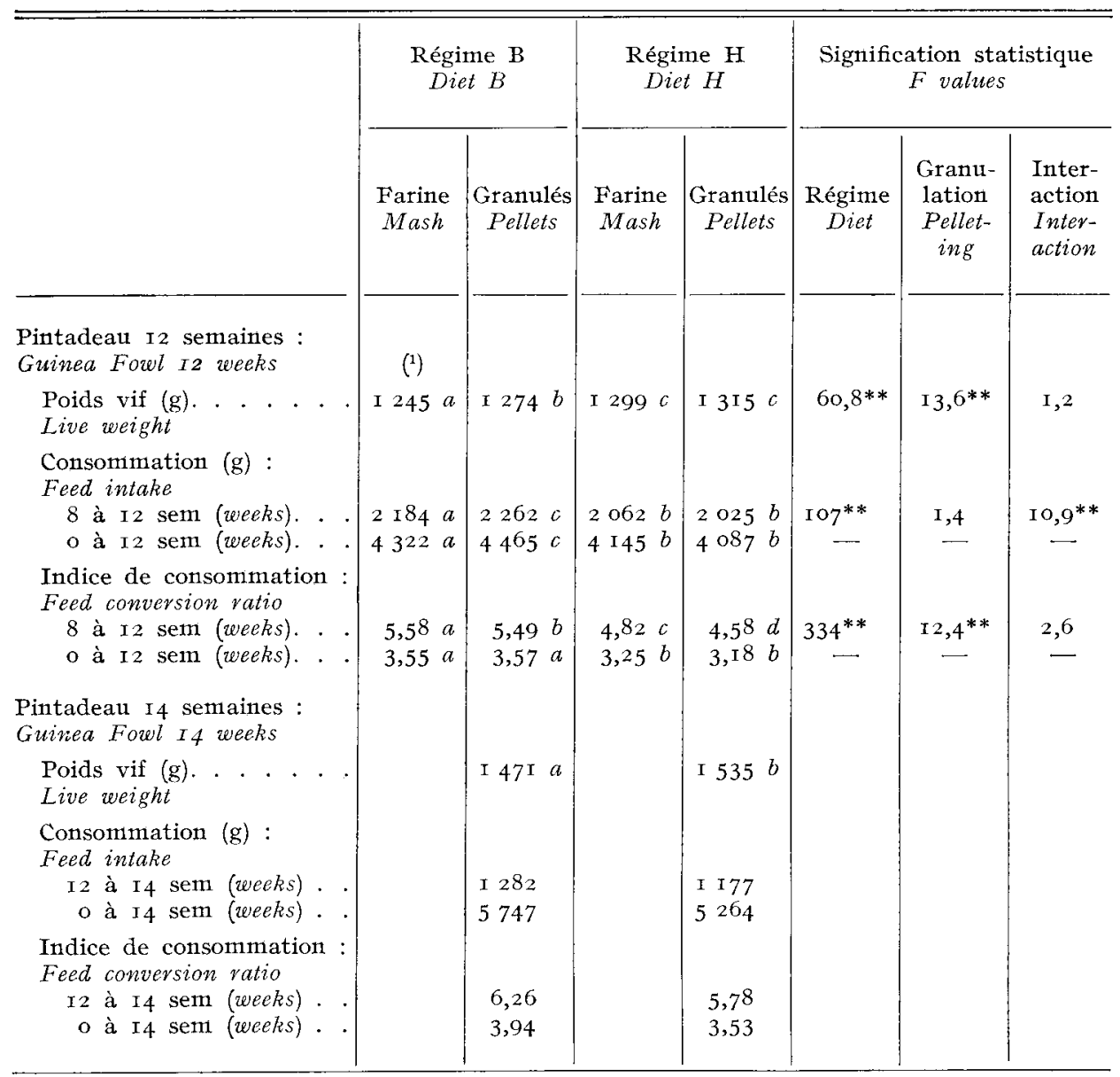

(1) A chaque âge, les performances qui ne sont pas suivies de la même lettre diffèrent significativement entre elles (At each age, values that are not followed by the same letter are significantly different).

la différence étant accusée pour les lots recevant les régimes $H$. Le gras abdominal est toujours plus abondant chez les femelles que chez les mâles, il est augmenté par les apports énergétiques élevés et s'accroît avec l'âge des pintadeaux. Une étude antérieure nous ayant montré l'existence d'une relation linéaire entre le gras abdominal et les lipides corporels (BLUM et LECLERCQ, I978), nous avons calculé la quantité totale de lipides entrant dans la composition du poids vif. Bien entendu, les mêmes facteurs, qui sont responsables du dépôt des graisses 
TABLEAU 5

Poids vif et état d'engraissement des pintadeaux mâles et femelles disséqués aux âges de $\mathrm{I}_{2}$ et $\mathrm{I}_{4}$ semaines

Live weight and fattening of guinea fowl according to sex, diet or age

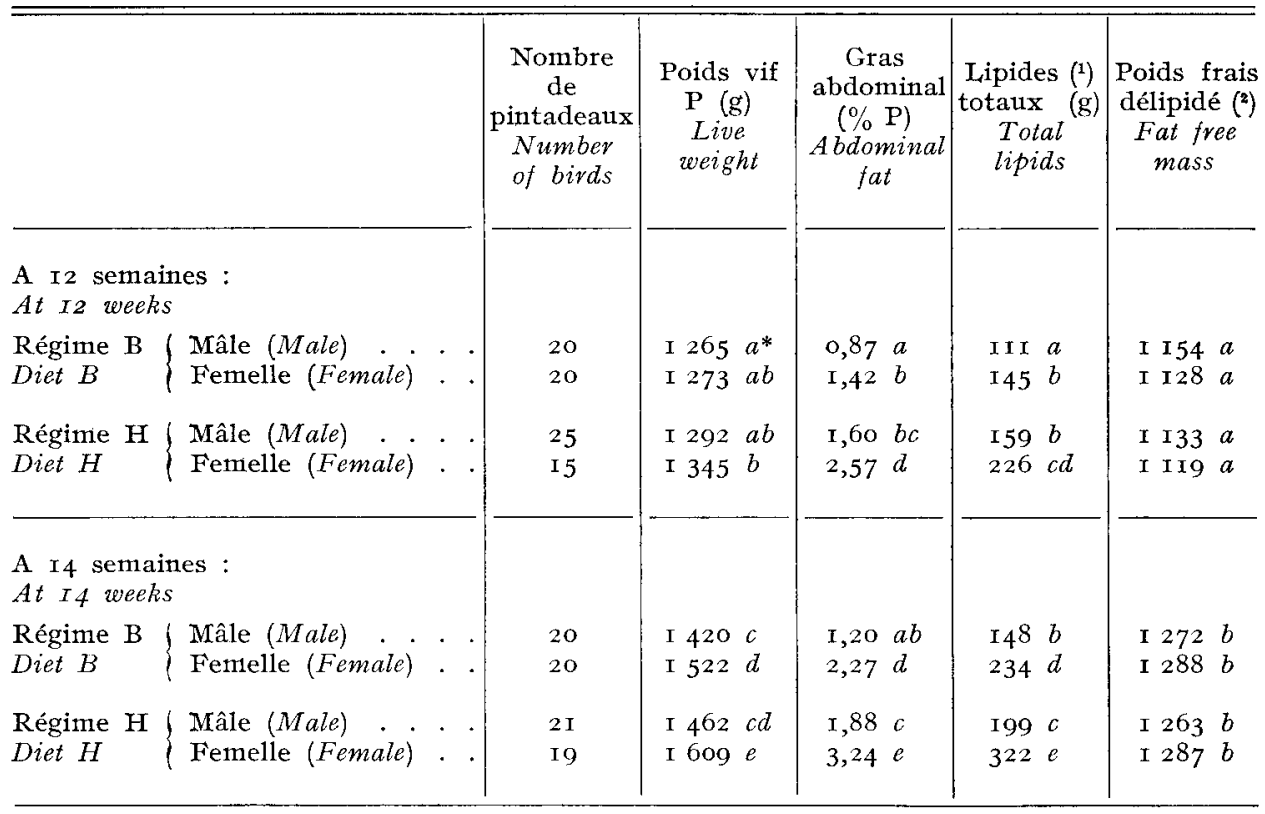

(1) Calculés à partir de la relation : lipides (p. roo) $=$ gras abdominal (p. roo) $\times 4,2+6,3$ (Calculated from the relation: lipids $(p . I 00)=$ abdominal fat $(p$. IOo $) \times 4.2+6.3)$.

( $\left.{ }^{2}\right)$ Poids frais délipidé $=$ poids vif - lipides totaux (Fat free mass $=$ live weight — total lipids).

* Dans chaque colonne, les valeurs qui ne sont pas suivies de la même lettre diffèrent significativement entre elles (In each column, values that are not followed by the same letter are significantly different).

abdominales, contribuent à accroître les lipides totaux : sexe femelle, âge et niveau énergétique. Après déduction des lipides totaux, il reste un ensemble de composants corporels qui forment la "masse maigre " ou "poids frais délipidé ". On constate que le poids frais délipidé augmente avec 1'âge, mais ne dépend ni du sexe, ni du niveau énergétique de la ration.

\section{Discussion}

Chez le poulet, nos observations rejoignent celles de nombreux auteurs. La granulation facilite l'ingestion et réduit le gaspillage; elle accroit les performances surtout lorsque le niveau énergétique de la ration est bas (CALET, I965). L'effet bénéfique de la granulation peut d'ailleurs disparaître totalement lorsqu'on incorpore au régime une forte quantité de graisse (SELL et ThOMpson, I965).

Chez le pintadeau, nous n'avons connaissance d'aucune étude systématique 
sur les effets de la granulation. Seuls, MEvEL et Bougon (I966) ont fait varier la présentation du régime en même temps qu'ils modifiaient sa composition. Ces changements étant associés, il est difficile de tirer de leurs travaux des conclusions certaines. Cependant, leur constation que farine, miettes ou granulés assurent les mêmes performances pendant la période de démarrage (o-28 jours) vont tout à fait dans le sens de nos propres observations. L,e très jeune pintadeau assure la réplétion de ses réservoirs digestifs indépendamment de la qualité de l'aliment. Cela explique sa sensibilité aux variations de l'apport énergétique pendant la période de démarrage, sensibilité déjà observée au cours de nos essais antérieurs (BLUM, GUILLAUME et LECLERCQ, I975).

Pendant les périodes de croissance et finition, MEver, et Bougon (I966) ont conservé seulement deux types de présentation : miettes et granulés. Ils obtiennent un même poids vif, mais des indices de consommation différents. Une partie de l'aliment distribué sous forme de miettes serait perdu par gaspillage avec pour conséquence une augmentation de l'indice. Dans notre essai, nous avons pris le maximum de précautions pour limiter le gaspillage. Le bénéfice apporté par la granulation ne concerne d'ailleurs pas uniquement l'indice, mais aussi le poids vif des pintadeaux qui ont consommé les régimes $B$ de $o$ à $\mathbf{I} 2$ semaines. Il n'y a pas opposition entre le pintadeau et le poulet. Mais, la période de démarrage passée, le premier a des besoins énergétiques plus stricts. Il ajuste au mieux sa consommation pour les satisfaire. Ainsi, il surconsomme à peine après granulation et réduit son ingestion alimentaire lorsque le niveau énergétique augmente.

L'analyse des constituants corporels rend compte des réactions du pintadeau. Dans une étude antérieure, nous avons montré que le gras abdominal reflète l'importance de l'engraissement. Le coefficient de corrélation entre gras abdominal et lipides corporels est de 0,95 (BLUM et L,ECI,ERCQ, I978). Les graisses constituant de simples réserves, on peut estimer à la suite de DELPECH (I966) que le poids frais délipiđé mesure le développement réel de 1'individu. Or, il apparaît que ce poids frais délipidé ne dépend ni des régimes consommés, ni du sexe, mais seulement de l'âge des pintadeaux. Autrement dit, les facteurs qui augmentent le gain de poids à un âge donné agissent par un simple accroissement des dépôts adipeux. Les régulations qui interviennent pour réduire l'appétit sont celles qui, chez l'adulte, assurent un état d'engraissement à peu près stable. La différence entre mâle et femelle peut être rapportée à 1'activité sexuelle, 65 p. Ioo des femelles ayant un ovaire hypertrophié à l'âge de I4 semaines.

En conclusion, à condition de veiller au gaspillage, on peut se dispenser de la granulation des régimes distribués au pintadeau. Si les apports énergétiques élevés accélèrent légèrement la croissance, c'est, en dehors de la période de démarrage, par stimulation exclusive de la lipogenèse. Sans contrôle de l'engraissement, la recherche d'un poids vif élevé à I 2 et I4 semaines pourrait aboutir à la production de pintadeaux trop gras.

Accepté pour publication en juin 1979.

\section{Summary}

Influence of energy level and pelleting of diets

on growth and fattening performances of guinea fowl as compared with chicken

A factorial experiment including 4 groups according to the energy level $(2,900$ to $3,240 \mathrm{Kcal}$ M.E. $/ \mathrm{kg}$ ) and the physical form (mash or pellets) of the diet, was carried out.

Each group included 6 cages of chicken (53 chicken per cage) subjected to the experiment 
from 7 to 56 days of age and 6 cages of guinea fowl (62 guinea fowl per cage), tested from the age of 7 to 84 and 98 days.

The favourable effects of pelleting were confirmed in chicken. These effects on the performances (live weight gain, feed conversion ratio) were more marked with the low energy diets. In guinea fowl the feed intake was the same in all groups at the age of 28 days. But thereafter the animals adjusted their consumption according to the energy level so that the influence of the two factors studied on weight gain was lower than in chicken. At the age of 12 weeks, pelleting had to significant effect on the live weight of guinea fowl receiving the high energy diet and only a small effect $(\div 30 \mathrm{~g})$ in those fed the low energy diet.

Carcass examination showed that the factors increasing the live weight of guinea-fowl aged 12 and I 4 weeks (high energy level of the diet, sexual maturity in the females) act exclusively by increasing the fat depots.

\section{Références bibliographiques}

Blum J. C., Guillaume J., Leclerce B., I975. Studies of the energy and protein requirements of the growing guinea-fowl. Brit. Poult. Sci., 16, I57-168.

BIUM J. C., LECLERCQ B., I978. Influence de l'alimentation, de l'âge et du sexe sur la composition corporelle du pintadeau in La composition corporelle des volailles, I.N.R.A. Rech. Avic. edit., Nouzilly, 37380 Monnaie (France), I 7-26.

Cavchard J. C., I97. La pintade, i vol., I re édit. H. Peradon, Uzès (France).

CALET C., I965. The relative value of pellets versus mash and grain in poultry nutrition. $W$. Poult. Sci. J., 21, 23-52.

DELPECH P., 1966. Le poids frais délipidé chez Gallus gallus : relations qui unissent ces constituants. C. R. Acad. Sci., 263, I 735-1 738 .

KEULS M., I 952 . The use of the studentized range in correction with analysis of variance. Euphytica, 1, II2-I 22.

MFvel M., Bougon M., I966. Étude des performances de différentes variétés de pintadeaux. Bull. Ploufragan, 6, 5-8.

SELL J. C., THOMPSON O. J., I965. The effects of ration pelleting and level of fat on the efficiency of nutrient utilisation by the chicken. Brit. Poult. Sci., 6, 345-354. 